\title{
POSIBILIDADES DE INTERVENCIÓN EN EL QUEHACER COTIDIANO
}

Germán Pacheco Borrella

Enfermero Psiquiátrico

Jerez de la Frontera (Cádiz)

Antonio Porras Cabrera

Concepción Bilbao Guerrero

Correspondencia:

$P^{a}$ de las Delicias, núm. 41, portal 2, $1^{a} \mathrm{C}$

11406 Jerez de la Frontera.

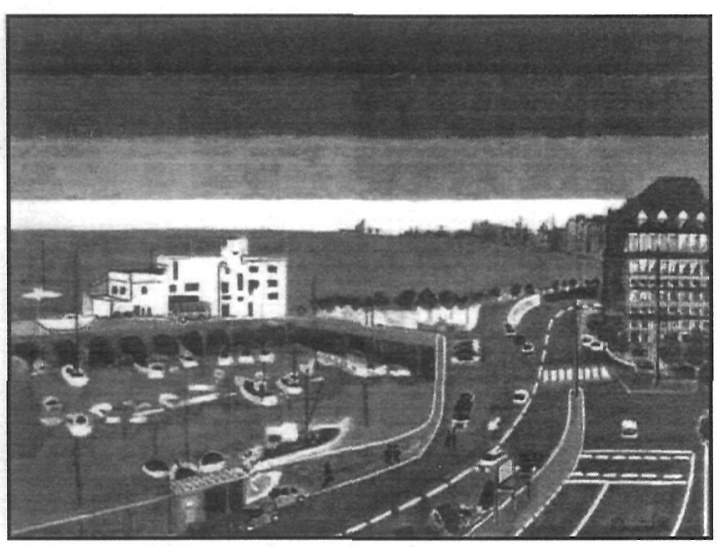

Javier de la Fuente

\section{RESUMEN}

El quehacer cotidiando de la enfermera de salud mental está condicionado por el ámbito donde desarrolla su tarea, por la legislación que regula la atención a la salud mental y por la evolución de la enfermería psiquiátrica y de la enfermería en general. Se describen a continuación algunas de las posibilidades de intervención que se tienen en el quehacer de la práctica cotidiana; y se finaliza abogando por un cambio actitudinal en el colectivo enfermero que posibilite enrraizar la profesión en la comunidad y a la par ejercer acciones de autocuidado sobre sí mismo como profesionales.

\section{POSSIBILITIES OF ACTION IN NORMAL DAY-TO-DAY WORK}

\section{SUMMARY}

The normal day-to-day work of mental health nurses is limited by the environmental area where they work, by the local and national regulations which rule mental health care and by the development of psychiatric nursing and general nursing rules themselves. Certain possibilities of taking action in the normal day-to-day work are here described.

This work suggests a proposal for an attitudinal change in the nursing body which will promote a link with the mental health profession in the community, improving their self-consciousness as professionals. 


\section{INTRODUCCIÓN}

Antes de plantear algunas de las posibilidades de intervención que las enfermeras y enfermeros de salud mental tenemos en nuestro quehacer cotidiano -posibilidades que pueden ser infinitas o limitadas-, deseamos compartir con el lector algunas reflexiones previas que tienen que ver con los contextos donde nos desenvolvemos habitualmente los profesionales de la Enfermería, con el marco legal que da soporte al nuevo modelo de atención a la salud mental y con la evolución del colectivo que hemos podido apreciar de forma más concreta en la última década.

Probablemente coincidamos en que existen, fundamentalmente, dos contextos bien diferenciados en los que se desarrolla y desenvuelve a diario la enfermera psiquiátrica: uno es el Hospital Psiquiátrico (H.P.), lugar donde tradicionalmente ha venido "atendiéndose" y enclaustrándose la locura. El otro es el Sistema Nacional de Salud (S.N.S.), donde se ubican los dispositivos comunitarios de atención a la salud mental, que configuran la red alternativa de aquél. Y dentro del S.N.S., podríamos distinguir dos espacios también distintos: el hospitalario y el comunitario. Y dentro del ámbito comunitario distinguiríamos, porque en buena parte son distintas, las intervenciones en espacios tan diferenciados como son la consulta de enfermería y el domicilio del ciudadano.

El contexto, en definitiva, va a condicionar sustancialmente el tipo de intervenciones enfermeras en la práctica cotidiana y va a conferir unas determinadas características al quehacer enfermero que, en conjunto, contribuyen a delimitar en buena medida el perfil profesional de la enfermera de salud mental.

Existe otro condicionante, no menor en importancia, que ha tenido y sigue teniendo una gran repercusión en la práctica del colectivo enfermero en general y en el de salud mental en particular. Esto es: el marco legal.

La legislación vigente, ha hecho posible que la enfermera aplique sus conocimientos y habilidades en su quehacer y ejerza la relación de ayuda con cuantos ciudadanos, sanos y/o enfermos, usan los servicios sanitarios. Como ejemplo, podríamos señalar muy diversas situaciones. No obstante, valga decir que no es lo mismo ejercer en el ámbito de la atención primaria de salud, que en el modelo -llamémosle- "tradicional". Es decir, que no es lo mismo trabajar en un Centro de Salud, en el seno de un equipo interdisciplinar, prestando cuidados y realizando actividades orientadas a la promoción, protección y rehabilitación de la salud, que en un típico Ambulatorio siendo "A.T.S." de zona, dedicado básicamente a la aplicación de tratamientos reparadores.

Centrándonos en nuestra parcela sanitaria, recordemos que el marco legal de la Reforma Psiquiátrica es el artículo 20 de la Ley General de Sanidad (14/86, de 25 de abril), que es la respuesta normativa fundamental al mandato constitucional que consagra el derecho que tienen todos los ciudadanos a la protección de la salud. Sin embargo, el documento que orienta el desarrollo de esta Ley y sirve de base para la Reforma es el Informe de la Comisión Ministerial para la Reforma Psiquiátrica que se publicó en abril de 1985.

Ambos textos, constituyen lo que podríamos llamar un "Programa Marco" que teóricamente afecta a todos por igual, pero que en la práctica cada Comunidad Autónoma lo está desarrollando en "su" momento y a "su" manera. Y, por tanto, la Reforma está teniendo un lento y desigual desarrollo e implantación entre unas Comunidades y otras.

En el Informe de la Comisión Ministerial se explicitan las directrices generales de un modelo para la atención y asistencia a los problemas de salud mental de la población española; y pone de manifiesto el carácter comunitario que tienen que tener los dispositivos correspondientes; sin embargo, ni la Ley General de Sanidad ni las recomendaciones y propuestas de la Comisión Ministerial hacen mención expresa a los H.P.; aunque ésta última vagamente habla de su "superación". (1)

En la práctica, cada Comunidad Autónoma, partiendo de esos principios generales, ha elaborado "su particular" Programa de Atención a la Salud Mental; dándose una gran disparidad de criterios: tenemos desde Comunidades que no han iniciado la Reforma Psiquiátrica, pasando por otras que tratan de hacer coexistir el modelo benéfico y el modelo sanitario, hasta aquellas que -como Andalucía, Asturias...- han apostado por una Reforma que, 
además de contemplar el modelo de intervención comunitario o modelo biopsicosocial, se plantean como objetivo sustituir los H.P. por una red alternativa que de respuestas a las necesidades de atención a la salud mental de los ciudadanos. Y parece existir un consenso generalizado en denominar al nuevo modelo como alternativo, toda vez que con él se propugna que los nuevos servicios sirvan para hacer innecesarios los viejos y no para complementarlos o retroalimentarlos.

En este sentido, cuando se cierra un H.P., lejos de llorarlo -aunque bien es cierto que para algunos es imprescindible la elaboración del duelo por la "pérdida"-, nos congratulamos doblemente: por un lado, porque ello es indicativo de que se están cumpliendo los objetivos de la Reforma Psiquiátrica; y, por otro, porque significa para nuestra sociedad el abandono de formas arcáicas de relación con la locura; sobre todo si compartimos que las instituciones sociales -como el manicomio- son estructuras organizadas al servicio de las defensas antiansiedad en el colectivo y que, por tanto, la desaparición de una institución como la manicomial y su reemplazo por una red de estructuras alternativas, señala un avance del grupo social hacia formas más elaboradas y complejas de organización (2).

Por otro lado, aportemos alguna de las cuestiones que se ponen de manifiesto en el Informe Técnico núm. 105 de la O.M.S., que fue elaborado en su día por un grupo de expertos en Enfermería de Salud Mental: "La práctica de la enfermería psiquiátrica depende directamente de la actitud tradicional de la colectividad respecto de los enfermos mentales, de los progresos de la psiquiatría y de la evolución de la profesión de enfermería" (3).

Además del contexo donde se producen las intervenciones enfermeras y del marco legislativo, hay que hacer referencia a la evolución que ha tenido la Enfermería Psiquiátrica. En ese proceso dinámico y evolutivo hemos percibido una Enfermería Psiquiátrica que, hoy, se nos antoja caduca y acaba$\mathrm{da}$, que ha estado anclada en el manicomio, que ha funcionado sólo para la contención, la custodia y el direccionismo con el paciente. Una Enfermería añeja, que ha sido un poco de todo y nada de ella misma; que ha sido una Enfermería olvidada y marginada del resto de la profesión. Quizás se automarginara, pero también es posible que fuera ab- sorbida como tantas otras cosas por esa "Institución total" (Goffman, 1987) (4) que es el manicomio.

Sea como fuere, debemos asumir esa Enfermería porque de ella provenimos al igual que de una Enfermería general que, en España, ha pasado por múltiples vicisitudes: cambios de nombre, cambios en los planes de estudio, indefinición jurídica, falta de planificación, ausencia de una formación postbásica, etc.

Pero por suerte para el usuario y para esa "vieja" Enfermería, en muchas Comunidades Autónomas de España ha sido posible realizar un proyecto de Reforma Psiquiátrica, toda vez que se han dado las condiciones sociales, políticas y administrativas favorables para ello, concurriendo además con la existencia de conocimientos científicotécnicos que la han posibilitado. (5)

Y ello ha venido a coincidir con un despertar de la Enfermería española en general, que se inicia una década antes que la Reforma Psiquiátrica, y cuyo exponente más significativo fue la entrada en la Universidad. Entendemos que estos hechos han sido fundamentales para que la Enfermería Psiquiátrica española saliera del ostracismo y de la agonía en que estaba inmersa y evolucionara hacia la luz y la revitalización.

Pero además, ha favorecido su evolución la existencia de un conocimiento enfermero y la capacidad y habilidad de unos profesionales que han sabido responder a las necesidades del paciente-usuario y familia en un contexto más normalizado.

Por tanto, hay que poner de manifiesto que se ha producido una profunda transformación del rol profesional y hoy podemos decir que existe una nueva y joven Enfermería de Salud Mental y Psiquiátrica. $\mathrm{Y}$ desde ésta, es donde vamos a hacer referencia a esas posibilidades, quizás infinitas pero en ningún caso limitadas, que tenemos en nuestro quehacer cotidiano.

La Enfermería de Salud Mental se caracteriza por ser un servicio humano, al que le guía la filosofía humanística, y cuyo cometido principal es la provisión de cuidados. Y sus ámbitos de actuación son aquellos en los que se halla el usuario del S.N.S.: equipos de salud mental en la comunidad, 
unidades de hospitalización en los hospitales generales, unidades de salud mental infantil, unidades de rehabilitación, comunidades terapéuticas, hospitales de día, pisos protegidos, etc.

La especificidad de cada servicio implica que la enfermera de salud mental realice acciones concretas y quehaceres asistenciales determinados; pero común a todos ellos podemos decir que cumple funciones preventivas, asistenciales, terapéuticas y rehabilitadoras.

La nueva Enfermería de Salud Mental y Psiquiátrica interactúa -a través de las enfermeras profesionales- con otras disciplinas -representadas por otros profesionales- en el espacio interdisciplinar que constituye el equipo de salud mental. Pero también tiene un espacio propio: la provisión de cuidados. Como dice Orem (1980) la enfermera actúa cuando un individuo, familia o grupo no son capaces de satisfacer sus requisitos de autocuidado, porque no saben, no pueden o no quieren, y por tanto interviene enseñando, ayudando-colaborando, supliendo. (6)

De otro modo, también podemos decir que, en todo individuo, cuando la exigencia para el autocuidado terapéutico es mayor que la capacidad que posee para proveerse el mismo, surge la demanda de cuidado enfermero; cuidado que es una acción intencionada realizada por personas concretas de un grupo social -las enfermeras y los enfermeros- para ayudar a otros que presentan déficits en autocuidados. Por eso, el servicio enfermero es un servicio de ayuda.

Una vez planteado este marco conceptual, conviene enfatizar que no existe una Enfermería para "agudos" y otra para "crónicos", ni hay una Enfermería para la "neurosis" y otra para la "psicosis", y tampoco entendemos que exista una Enfermería de Salud Mental "manicomial" y otra "comunitaria". Sólo existe la Enfermería de Salud Mental y Psiquiátrica como servicio humano, como ciencia y como arte para satisfacer las necesidades de autocuidado terapéutico presentes y futuras.

Por cuanto hemos expuesto hasta aquí, las posibilidades de intervención que tenemos en nuestro quehacer cotidiano si no infinitas, son muchas las probables: proveer el cuidado, capacitar y habilitar, promover la autonomía, educar para la salud, favorecer el desarrollo de las personas, promover su socialización; luchar contra la marginación del enfermero mental, fovoreciendo su integración en la comunidad, etc.

Ya hemos dicho -y parece obvio-, que el quehacer viene determinado por el ámbito donde prestamos nuestros servicios: no es lo mismo el quehacer en un maniocomio, que el quehacer en el medio comunitario.

Con la venia del lector, prescindiremos de hacer referencia a las posibles intervenciones en un $\mathrm{H}$. P., en donde entendemos que lo único posible, además de deseable, es propugnar su transformación, en el mejor de los casos, para proceder al desmantelamiento y cierre del mismo. Pues no concebimos que hoy, en los albores del siglo XXI, persistan "hospitales" monográficos a la antigua usanza -lazaretos- separados del S.N.S. Si se admiten, se corre el riesgo de ver muy pronto un lugar de segregación para los "sidosos", por ejemplo. Hoy, desde el conocimiento y la razón y en una sociedad democrática, no se concibe que haya que perder la libertad por padecer un trastorno mental. ¿Quién de nosotros no tiene a nadie próximo afecto de un trastorno emocional? Un compañero de trabajo nos decía hace poco tiempo: "Sin ir más lejos, un familiar mío padece de "los nervios" y tiene "depresiones", mi mejor amigo es un psicótico que trabaja en una entidad bancaria, una compañera de trabajo está en tratamiento por padecer una crisis de ansiedad generalizada con estado de ánimo deprimido, y uno mismo a buen seguro tiene filias y fobias, unas veces manifiestas, otras ocultas. ¿Por mor de qué deberíamos estar enclaustrados en un manicomio y alejados del sistema sanitario, de nuestras familias, trabajo, amigos..?"

Por consiguiente, nos vamos a centrar en un quehacer cotidiano en el ámbito comunitario, que es el lugar natural del usuario al que prestamos nuestros servicios. Tengamos en cuenta que para el quehacer enfermero hay que pensar en "Enfermería". Esto implica una acción intencionada, y requiere un cambio actitudinal. También implica tener conocimientos enfermeros. Y además, se requiere, entre otras cosas, que, en lo cotidiano, en ese quehacer pequeño o grande, debemos traslucir qué somos, quiénes somos. 
Así tendremos la posibilidad, de entre las muchas en nuestro quehacer, de saber escuchar, saber estar y saber dar respuestas cuando estemos frente a un padre o una madre que manifiesta alta emoción expresada y tiene un alto nivel de criticismo hacia su hijo, porque no entiende nada de cuanto le sucede; y eso es hacer Enfermería.

Estará la posibilidad de intervención frente a un neurótico obsesivo/compulsivo y hacer que afronte eficazmente los hechos de la vida cotidiana, eleve su autoestima y se exprese asertivamente; y eso también es hacer Enfermería.

Esa posibilidad de contener ansiedades individuales y familiares; esa posibilidad de nombrarse enfermero para ser reconocido por el "otro", y compartir el conocimiento enfermero con éste para que un día demande cuidados urgentes, es hacer Enfermería.

Plantearse y ejecutar una intervención psicosocial, familiar, en un caso de distimia y conseguir dar un vuelco a la evolución del caso, por supuesto, es hacer Enfermería.

Reforzar social y positivamente a una esposa por su saber hacer y por su implicación en el plan de cuidados de su marido psicótico, también es un quehacer enfermero.

Como igualmente lo es, coordinarse interdisciplinarmente en los espacios comunes del equipo aportando nuestros conocimientos enfermeros $y$ nuestro saber hacer.

Administrar un fármaco inyectable, cuando el paciente/usuario no puede hacerlo por sí mismo; implicar a un paciente en su proceso de autocuidado y ayudarle a superar dificultades -como por ejemplo, frente a reacciones de pánico y fuga-; trabajar las habilidades sociales de forma individual y/o grupal, son otros quehaceres enfermeros.

Esa posibilidad que tenemos de construir y desarrollar una experiencia grupal para los autocuidados, con neuróticos o psicóticos; esa otra de redactar un Informe de Enfermería que acompañe al paciente de un dispositivo a otro de la red; o la de disponer de un instrumento para la valoración de los déficits de autocuidado y trabajar con un lenguaje, modelo y/o teoría común; o aquella que consiste en coordinarse con la enfermera de atención primaria para hacer efectiva una atención integral y/o establecer un programa de consulta de enfermería para psicóticos crónicos en ese ámbito... Todo esto también es Enfermería.

Coordinarse con Instituciones sociales y establecer programas de intervención y/o cooperación; así como promocionar la salud mental en el medio comunitario y, por ejemplo, elaborar un programa de educación para la salud mental en las Escuelas; o diseñar y ejecutar el programa de un grupo de relajación para usuarios con neurosis; por supuesto, también es hacer Enfermería.

Garantizar la continuidad de cuidados, mientras el usuario permanece en la red de atención a la salud mental del S.N.S., es, además, un deber de las enfermeras de salud mental. Etcétera, etcétera...

Antonio Gala dijo en cierta ocasión: "Mi mayor esperanza es el ser humano, porque es capaz de hacer las más bellas cosas". (7) Y, efectivamente, creemos que los psicóticos y los neuróticos son seres humanos capaces de hacer las más bellas cosas. Sólo requieren que profesionales de la salud como los enfermeros y las enfermeras de salud mental, sean capaces de quehaceres cotidianos para su atención.

En definitiva, todo lo planteado hasta aquí, es parte de la realidad con la que nos acercamos al cambio de milenio. Y además, cabría preguntarse: ¿qué podemos hacer las enfermeras y los enfermeros de cara al futuro? Pues mucho, sin duda alguna. Permítasenos decir que abogamos, fundamentalmente, por promover un cambio actitudinal en el colectivo enfermero de salud mental que posibilite:

- Quehaceres infinitos en la cotidianidad de la práctica.

- Que el profesional de Enfermería, en un futuro inmediato, sepa nombrarse y carezca de conflictos de identidad que puedan psicotizarle.

- La aparición de liderazgos individuales, es decir, profesionales capaces de motivar y construir un discurso enfermero y que generen corrientes de opinión; y liderazgos colectivos -o sea grupos profesionales como la Asociación de Enfermería en Salud Mental o la Asociación Española de Enfermeras 
Docentes, por ejemplo-, que sean capaces de aglutinar voluntades y construyan un proyecto enfermero común y que eleven a la Enfermería al lugar que le corresponde en el contexto cultural, científico, social y político que nos está tocando vivir.

- Que se investige más y mejor para seguir ampliando el conocimiento enfermero y poder dar respuestas científicas a las necesidades, incapacidades y déficits que presente el ser humano o familia o grupo a quienes atendemos.

- La creación de un servicio enfermero que de respuestas permanentes y haga aportaciones para la atención de la locura y dejemos de ver el trastorno mental como algo mágico, temeroso, molesto, peligroso, fóbico...; porque de lo contrario volveremos a la contención y custodia.

- Que la enfermera de salud mental sea capaz de promover el cuidado; lo cual significa también hacer aportaciones y tener voz y voto en la determinación de políticas sanitarias, por un lado; y por otro, promocionar el cuidado del ser humano de manera inequívoca y siendo capaces de emitir opiniones ante acontecimientos sociales que afectan al vivir cotidiano de la ciudadanía.

- Promover y promocionar un modelo de provisión de cuidados cuyo fin sea transmitir nuestro saber al usuario para contribuir al desarrollo de personas cada vez con mayores niveles de autonomía.

Quizás todas estas cosas y muchas más nos suenen a utópicas. Y es probable que lo sean parte de ellas. A pesar de lo cual, seguimos reivindicando el derecho a ser utópico. Siempre hemos creido y seguimos creyendo que sin la utopía no se avanza. Y sabido es, que cosas que ayer fueron utópicas, hoy son una realidad.

Por tanto, animo a todos a dejar de ser un colectivo silencioso socialmente y a hacer que la voz de las enfermeras de salud mental se haga oir y escuchar frente a los problemas sociales, e incidamos en las deciones que tienen que ver con nuestro ámbito profesional. Y concluimos con un deseo: la enfermera de salud mental, en el futuro inmediato, deberá tener conocimiento, capacidad y motivación para ejercer acciones de autocuidado y alcanzar el máximo grado posible de madurez y desarrollo profesional y científico; o lo que es lo mismo: la enfermera de salud mental y psiquiátrica debe autocuidarse.

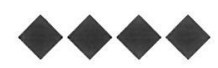

\section{BIBLIOGRAFIA}

1.- Pacheco Borrella, G; Vila Blasco, B. (1994) Sobre los programas de salud mental en España. Ponencia presentada en las Jornadas Internacionales de Salud Mental, organizadas por la Asoc. de Enfermería en Salud Mental y la Federación Mundial de Salud Mental, Madrid.

\section{2.- GRUPO DE TRABAJO PROVINCIAL:} Hospital Psiquiátrico Provincial: Proceso de desmantelamiento. Dispositivos intermedios. Documento elaborado por un grupo de profesionales de la provincia de Cádiz, constituidos en Comisión Técnica Asesora, para orientar técnicamente sobre el desmantelamiento del H.P. de El Puerto de Santa María. Documento no publicado.

3.- (O.M.S) (1956) Serie Informes Técnicos núm. 105. Comité de expertos en enfermería psiquiátrica. Ginebra.

4.- Goffman, E. (1987) Internados. AmorrurtuMurgía, Madrid.

5.- Carmona Calvo, J. (1995) Atención a la cronicidad, tras el cierre del Hospital Psiquiátrico". Ponencia presentada en la Jornada de Salud Mental: Atención a la cronicidad psíquica. Organizada por la Asoc. de Enfermería en Salud Mental, Jerez de la Frontera (No publicada).

6.- Orem, D. (1993) Modelo Orem. Conceptos de enfermería en la práctica Ed. Científicas y Técnicas. Masson-Salvat, Barcelona.

7.- Gala, A. (1995) Entrevista, concedida a el períodico EL MUNDO ( 4 de marzo de 1995). 